\section{"U Can't Touch This" With Pharmacotherapy Alone for Weight Loss or Smoking Cessation}

The 2 outcomes-achieving weight loss in overweight persons and tobacco cessation in smokers-have interesting parallels in the absolute and relative value of pharmacotherapy. Both smoking and obesity are established risk factors for cardiovascular disease, while, individually, smoking is associated with chronic obstructive pulmonary disease (COPD) and obesity with diabetes. COPD and diabetes have been selected by the Centers for Medicare and Medicaid Services (CMS) as 2 of the 3 highest expenditure disease conditions to be targeted for disease management in the Chronic Care Improvement Program (CCIP) of the Medicare Modernization Act of 2003. ${ }^{1}$ Both smoking and obesity also are controllable, having clear but not easy solutions.

Smoking is the most common cause of COPD, responsible for $80 \%$ to $90 \%$ of all COPD deaths. Unfortunately, the majority of smokers who likely have COPD ignore their symptoms, contributing to the total of 120,000 Americans who die each year from COPD. ${ }^{2}$ In a previous issue of JMCP, Tinkelman et al. reported that COPD is the only chronic illness where the morbidity and mortality rates are going up, as are the direct and indirect costs of the disease. ${ }^{3}$ More than 1.7 million Medicare beneficiaries had a diagnosis of COPD for claims paid through June 2004. The Medicare beneficiary with COPD accounts for mean annual expenditures that are about 2.4 times the average for all Medicare beneficiaries. ${ }^{4}$ It is no wonder that CMS identified COPD as one of the 3 most important targets of disease management in CCIP.

Besides CMS recognizing tobacco-related disease burden, the association with higher direct (medical) and indirect (e.g., workplace) costs has not been lost on employers. Some employers are screening job applicants to prevent hiring persons who smoke, and others are forcing current employees who do not quit smoking to leave their jobs. ${ }^{5}$ Alaska Airlines has for almost a decade required applicants to pass a urine test for tobacco in order to be considered for employment, while Union Pacific Corp. (Omaha, NE) in the fall of 2004 stopped hiring smokers in certain states, including Arkansas and Texas. ${ }^{6}$ Other employers require smokers to pay more for their health care coverage. In July 2005, Navistar International Corporation (Warrenville, IL), a large truck manufacturer, began charging $\$ 50$ more per month for health care coverage to employees who smoke. Beginning January 1, 2006, employees of Northwestern Mutual Life Insurance Co. (Milwaukee, WI) will be subject to a $\$ 25$ fee on monthly health care premiums if the employee or a dependent smokes. In the public sector, the state of Georgia began charging each employee a premium surcharge of $\$ 40$ per month on July 1, 2005, if the employee or a dependent is a smoker, regardless of frequency of use. ${ }^{7}$ For Alabama employees, the surcharge is \$20 per month, beginning October 1, 2005. South Dakota has assessed a $\$ 30$ premium surcharge for smokers since 1997. State employees and retirees in Pennsylvania are required to sign a "Tobacco Affidavit" certifying that they are tobacco-free to obtain discounts on health and life insurance premiums.

Various branches of the federal government have made efforts to help U.S. citizens quit tobacco use. U.S. Department of Health and Human Services (HHS) Secretary Tommy G. Thompson announced on December 23, 2004, that CMS intended to provide new coverage allowing certain Medicare beneficiaries who smoke to receive cessation counseling services. ${ }^{8}$ The HHS announcement for Medicare coverage did not include coverage for pharmacotherapy. U.S. Internal Revenue Service (IRS) ruling 99-28 in June 1999 revoked a 20-year-old regulation that limited the tax deductibility of smoking cessation programs to employees with specific ailments such as emphysema. The new ruling made the costs of smoking cessation programs, including prescription drugs, tax deductible even if the individual employee did not have a specific smoking-related disease. These health benefits are tax deductible and can be funded through pretax contributions to flexible spending accounts (FSAs). The IRS ruling was influenced by the growing evidence that smoking is a clear and direct threat to health. The ruling said "Scientific evidence has ... established that nicotine is addictive and that smoking is detrimental to the health of the smoker." On July 23, 2004, the U.S. Department of the Treasury defined smoking cessation drugs in the same category as statins, angiotensin-converting enzyme inhibitors, and weight-loss drugs as exempt from the $\$ 1,000$ annual deductible in the new health savings accounts (HSA) established under the Medicare Modernization Act of 2003. "Drugs or medications are preventive care when taken by a person who has developed risk factors for a disease that has not yet manifested itself or not yet become clinically apparent ... or to prevent the reoccurrence of a disease from which a person has recovered."10

In drug benefit plans, smoking cessation drugs have historically been excluded from most policies. Over the 3-year period from 1997 through 1999, about two thirds of employer-sponsored drug benefit plans excluded coverage for smoking cessation drugs. ${ }^{11}$ Nicotine replacement gum and transdermal patches are available over the counter (OTC), and coverage in drug benefit plans would be indicative of health plan support of smoking cessation by this method; however, OTC coverage by health maintenance organizations appears to be little changed, at 31.5\% of HMOs in 2003 compared with $32.1 \%$ in 2002 and $32.4 \%$ in $2001 . .^{12}$

Given the morbidity recognized by government and employers, why don't more payers cover cessation pharmacotherapy? The cost-effectiveness of pharmacotherapy has been firmly established. The U.S. Surgeon General's report from 2000 states, "Cost-effectiveness analyses have shown that smoking cessation treatments compare quite favorably with routine medical interventions such as the treatment of hypertension and 
hypercholesterolemia, and with other preventive interventions such as periodic mammography. In fact, smoking cessation treatment has been referred to as the 'gold standard' of preventive interventions." 13

The Cochrane Collaboration Group has published several reviews on the benefit of pharmacotherapy for smoking cessation. Regarding nicotine replacement therapy (NRT), all routes of drug delivery were effective when 103 studies were reviewed. The pooled odds ratio (OR) for abstinence at 6 months with NRT compared with control was 1.77 (95\% confidence intervals [CI], 1.66-1.88). The NRT benefit was seen regardless of additional support provided to the smoker. ${ }^{14}$ Certain anti-depressants are also considered first-line and have ample evidence. When used as the sole pharmacotherapy, bupropion (19 trials, OR 2.06; 95\% CI, 1.77-2.40) and nortriptyline (4 trials, OR 2.79; 95\% CI, 1.70-4.59) both doubled the odds of cessation at 6 months. ${ }^{15}$ The effect was seen even in the absence of clinical depression.

A more recent randomized controlled trial (RCT) conducted in 2004 after publication of the U.S. Clinical Practice Guideline reinforced prior evidence and supported nortriptyline in combination with NRT. The smoking cessation rates at 6 months were $23 \%$ for nortriptyline $75 \mathrm{mg}$ versus $10 \%$ for placebo; both groups received nicotine and behavioral therapy (absolute difference, 13\%; 95\% CI, 1.3\%-24.5\%; $P=0.052$ ). ${ }^{16}$ In the world of RCTs of pharmacologic interventions for smoking cessation, a $13 \%$ effect is not trivial and means that only 8 patients needed adjunct antidepressant therapy for one additional patient to successfully quit. Studies evaluating bupropion as an adjunct to NRT have had mixed results. ${ }^{17}$

Increasing the level of health insurance coverage or reducing direct costs of smoking cessation treatment to patients has been shown to be cost effective. In a recent Cochrane review of 6 controlled trials evaluating financial incentives for smokers, the pooled OR for achieving abstinence at 6 months was 1.48 (95\% CI, 1.17-1.88). The prolonged self-reported abstinence rate showed an absolute increase of $2 \%$ (95\% CI, 0.00-0.05). Although 2\% may seem small, costs per quitter varied between $\$ 260$ and $\$ 2,330 .{ }^{18}$ Other evaluations by the Agency for Health Care Research and Quality (AHRQ) calculated that costs per quality-adjusted life-years (QALYs) saved (1995 dollars) ranged from $\$ 1,108$ to $\$ 4,542$, if federal guidelines were implemented. ${ }^{19}$ A cost-effectiveness study from the Group Health Cooperative of Puget Sound showed that, with full coverage, "an estimated 2.8 percent of smokers stopped smoking per year, as compared with 1.3 percent with standard coverage, and 0.7 percent with reduced coverage. This increase in the annual rate of cessation among smokers with full coverage was achieved at a cost of $\$ 328$ per benefit user, which was clearly a bargain as compared with the average annual cost of medical treatment for hypertension $(\$ 5,921)$ or heart disease $(\$ 6,941)$, which is usually incurred for the life of the patient."20

Since smoking cessation efforts are cost effective, how are we doing with adoption of these measures? The results of managed care organization (MCO) interventions in smoking cessation are mixed. Measures used by the National Committee for Quality Assurance (NCQA) in the Health Plan Employer Data and Information Set (HEDIS) Medical Assistance with Smoking include 3 components: (1) the percentage of smokers or recent quitters who received advice to quit smoking from their practitioner, (2) the percentage of smokers with whom the practitioner discussed smoking cessation medications, and (3) the percentage of smokers whose practitioner discussed smoking cessation strategies. ${ }^{21}$ In the 2004 annual report from NCQA, commercial MCOs reported that $68.6 \%$ of current smokers or recent quitters received advice from practitioners to quit smoking in 2003, with a slightly lower rate for Medicare MCOs (63.3\%) and Medicaid MCOs (65.8\%). These rates have remained constant in the 4-year period from 2000 through 2003. Perhaps more important, only $37.6 \%$ of current smokers or recent quitters in commercial MCOs discussed smoking cessation medication with their practitioner in 2003, and only $36 \%$ discussed smoking cessation strategies. The 2004 NCQA report (for 2003 data) estimated the direct and indirect costs of smoking to exceed $\$ 157$ billion, or $\$ 3,443$ per smoker per year, and current smokers were associated with $18 \%$ higher health care costs over an 18-month period compared with those who never smoked.

If physicians have room to improve on compliance with promoting cessation, can pharmacists help fill the gap? Yes, they can. A Cochrane review looked at 2 RCTs from the United Kingdom that included 976 smokers, most of whom were using NRT. The community pharmacists used the Stages of Change counseling model to promote cessation. One study showed a significant difference in self-reported cessation rates at 12 months: $14.3 \%$ versus $2.7 \%(P<0.001)$; the other study showed a likely positive trend at each follow-up, with $12.0 \%$ versus $7.4 \%$ cessation $(P=0.09)$ at 9 months. ${ }^{22}$

Although pharmacotherapy for smoking cessation is a wise use of resources compared with widely accepted medications for cardiovascular conditions, how do weight-loss agents fare? Weight loss is often as difficult for obese persons as smoking cessation is for smokers. For clinicians caring for obese and overweight persons, clinical practice guidelines (CPGs) are evolving. The CPG from the American College of Physicians (ACP) in April 2005 was intended to complement the guideline of the U.S. Preventive Services Task Force. ${ }^{23}$ The ACP defined the target audience for its guideline as all clinicians caring for obese patients, defined as having a body mass index (BMI) of $30 \mathrm{~kg} / \mathrm{m}^{2}$ or greater.

The ACP 2005 guideline on the management of obesity in primary care states that (a) "Pharmacologic therapy can be offered to obese patients who have failed to achieve their weightloss goals through diet and exercise alone, but there needs to be a doctor-patient discussion of the drugs' side effects, the lack of 
long-term safety data, and the temporary nature of the weight loss achieved with medications before initiating therapy" (recommendation \#2) and (b) "For obese patients who choose to use adjunctive drug therapy, options include sibutramine, orlistat, phentermine, diethylpropion, fluoxetine, and bupropion. The choice of agent will depend on the side-effects profile of each drug and the patient's tolerance of those side effects" (recommendation \#4).

The ACP 2005 guideline also suggests that there are not sufficient data to determine if one drug is more efficacious than another. Using meta-analytical techniques, the pooled amount of weight lost with sibutramine was $4.45 \mathrm{~kg}$ at 12 months versus $2.89 \mathrm{~kg}$ at 12 months for orlistat, $3.6 \mathrm{~kg}$ at 6 months for phentermine, $3.0 \mathrm{~kg}$ at 6 months for diethylpropion, $3.15 \mathrm{~kg}$ at 12 months for fluoxetine, and $2.8 \mathrm{~kg}$ at 6 to 12 months for bupropion. A meta-analysis by Li et al., also published in April 2005, found equivocal results with fluoxetine, ranging from $14.5 \mathrm{~kg}$ of weight lost to $0.4 \mathrm{~kg}$ of weight gained at 12 or more months. ${ }^{24}$

The meta-analysis by Li et al. also found that (a) sibutramine with lifestyle modification was more effective than placebo with lifestyle modification in promoting weight loss in overweight and obese adults at all time points assessed (with an average of $4.5 \mathrm{~kg}$ more weight lost at 1 year in the sibutramine group, and patients taking sibutramine had a $20 \%$ to $30 \%$ greater likelihood of losing at least $5 \%$ of their body weight than did patients receiving placebo); (b) there was no evidence that sibutramine reduces mortality or morbidity from obesity-associated diseases; (c) systolic and diastolic blood pressure outcomes varied, with some studies reporting small decreases, and other studies reporting small increases; (d) fasting blood glucose level and hemoglobin Alc levels decreased slightly in sibutramine-treated patients, but there was no consistent effect on cholesterol or lipid outcomes; and (e) heart rate was consistently increased by about 4 beats/minute in patients who took sibutramine, but there were no deaths reported from adverse events. A subsequent letter to the editor ${ }^{25}$ criticized the meta-analysis for including the weight loss results for bupropion since that clinical trial's primary end point was depression, not weight loss; the weight loss was a side effect, not the intentional study outcome. ${ }^{26}$

While the amount of extra weight loss attributable to weightloss medications is modest ( $<5 \mathrm{~kg}$ at 1 year), the ACP 2005 guideline suggests that there might be hope in the association of weight loss and improved glucose tolerance and less progression to type 2 diabetes mellitus. "In other studies, similar amounts of weight loss positively influenced other obesity-associated cardiovascular risk factors, such as lipid levels and hypertension. Therefore, although trials of weight-loss drugs have not looked at these outcomes specifically, the benefits found with weight loss through diet and exercise may be applicable to the weight loss attained with medications." ${ }^{23}$

Despite the ACP guidelines and the meta-analysis, we still do not know (a) the value of combination drug therapy, (b) the percentage of weight regained after medications are withdrawn, (c) the long-term (>12 months) efficacy or safety of pharmacotherapy for weight loss, or (d) the incremental value of pharmacotherapy on obesity-associated risk factors such as lipid levels and hypertension. Combined, these factors hamper clinical decisions to continue drug therapy beyond 1 year.

In this issue of JMCP, Malone et al. apply a cost-effectiveness model to previously published research, the Long-term Outcomes of Sibutramine Effectiveness on Weight (LOSE Weight) study. ${ }^{27}$ LOSE Weight was a nonblinded, prospective, randomized, controlled trial involving overweight and obese patients enrolled in a weight-loss management program (WMP) operated by an HMO. ${ }^{28}$ The LOSE Weight trial enrolled adult patients aged 18 years or older between January 1999 and June 2000 who had either BMI $\geq 30 \mathrm{~kg} / \mathrm{m}^{2}$ or BMI of $27-29.9 \mathrm{~kg} / \mathrm{m}^{2}$ with one or more comorbidities, including diabetes, hypertension, and/or hyperlipidemia. The intervention consisted of subjects receiving a WMP with or without sibutramine, an inhibitor of norepinephrine and serotonin neuronal uptake.

WMP enrollees paid $\$ 240$ out of pocket to participate in this physician-directed, multidisciplinary program that included 5 monitored-care visits with a prevention specialist (\$20 each visit), attendance at 2 or more group-format weight management seminars, and participation in 20 American Heart Association (AHA) classes. The AHA courses included Slim for Life (\$70) and Active for Life (\$70); each program had 10 sessions. Patients enrolled in WMPs who were not study participants paid the same upfront costs. Study participants who completed 6 months of the study received a $\$ 50$ rebate gift check. Those patients who completed 12 months of the study received an additional $\$ 100$ check.

Results from the intervention were consistent with prior studies of sibutramine. The mean weight loss/percentage total body weight loss was significantly greater at 12 months in the sibutramine group (13.7 pounds, or $4.8 \%$ of body weight) than in the nondrug group (5.0 pounds, or $2.2 \%$ of body weight) $(P<0.001)$. Adding sibutramine to the WMP increased the total additional cost per patient by $\$ 44$ for each extra pound of weight lost beyond what WMP alone provided (95\% CI, 42-46).

A handful of points are notable about the LOSE Weight study and the follow-up pharmacoeconomic analysis performed by Malone et al. First, since the results of the LOSE Weight study were not published until 2004, it was not among the evidence considered by the ACP in its recent guideline regarding sibutramine. This point is notable since the ACP guideline suggests that weight-loss amounts gained from sibutramine may improve lipid and glucose markers, while the LOSE Weight study confirmed the findings in the meta-analysis by Li et al.lipid and glucose markers definitely did not improve after weight-loss medication, either at 6 or 12 months. Second, there was likely selection bias in the LOSE Weight study. A total of 
1,564 subjects were invited to participate in the study, but $976(62 \%)$ declined to participate because of lack of desire to be potentially enrolled in the control group. Only 588 potential subjects (38\%) agreed to take part. Third, although WMP was a lengthy behavioral modification program, participants' completion rate of the WMP and the threshold for inclusion in the study results was not presented. Sibutramine users only averaged 222 days of use. Would 365 days of compliance have changed the results? Fourth, there was an unequal dropout rate- $5.1 \%$ in the sibutramine group versus $24.7 \%$ in the WMP-only group. Fifth, the inclusion criteria resulted in the exclusion of patients who would otherwise be candidates for drug therapy intervention for weight loss in a typical MCO population, such as the exclusion of patients who were taking selective serotonin reuptake inhibitors, St. John's wort, triptan drugs, lithium, fentanyl citrate, or one of 6 other drugs. Sixth, Malone et al. did not find significant change in obesity-related or overall health care resource utilization and costs when comparing the preenrollment and postenrollment periods. Seventh, the authors were forced to use multiple regression analysis to interpret their data because the WMP-sibutramine and the WMP-only groups were not equivalent at baseline in key characteristics; weight, BMI, and waist circumference were significantly higher in the WMP-sibutramine group, and the WMPsibutramine group had higher percentages of patients in the BMI categories of $35-39.9 \mathrm{~kg} / \mathrm{m}^{2}$ and $\geq 40 \mathrm{~kg} / \mathrm{m}^{2}$.

Collectively, these limitations in the LOSE Weight study and the subsequent pharmacoeconomic analysis by Malone limit the reliability of the findings; the calculated Jadad score is only $2 .{ }^{29}$ On the other hand, since the magnitude of weight loss reported in the LOSE Weight study for sibutramine plus WMP is consistent with the findings from prior systematic reviews, their take-home points should not be summarily dismissed.

What do other researchers report on the cost-effectiveness of weight loss medications? One group in the United Kingdom was specifically formed in 1993 to help pharmacists and clinicians with these types of difficult decisions. The National Health Service (NHS) Health Technology Assessment (HTA) program publishes peer-reviewed systematic reviews when commissioned by the NHS. They have recently released 2 articles dealing with weight- loss medications. A 2002 report reviewed sibutramine and found pooled estimates from RCTs suggesting that sibutramine produced statistically significant greater weight loss than placebo at all observed end points out to 1 year (weighted mean difference for weight-change at 1 year: -4.1 to $-4.8 \mathrm{~kg}$ ). Of note, the cost per quality-adjusted life-year (QALY) was estimated as $£ 10,500\left(19,136\right.$ US\$). ${ }^{30}$

Another HTA report from 2004 reviewed the long-term effects and economic consequences of various obesity treatments. They estimated cost per additional life-year or QALY of no more than $£ 13,000$ (23,690 US\$). ${ }^{31}$ One group of UK researchers not associated with the HTA did a cost-utility model based on published RCTs. Factoring in the reduction of heart disease and diabetes associated with weight loss, they found the incremental cost per QALY of sibutramine was $£ 4,780$ (8,710 US\$). ${ }^{32}$

So are all these QALY values $<25,000$ US\$ worthy of our attention? If the usual U.S. cutoff for "effective" interventions is drawn at 50,000 US\$, then the answer should be yes. Particularly when combined with the burden of disease that obesity causes, even minor improvements can have significant public health benefits. However, since these cost analyses were based on earlier studies and more recent studies including the LOSE Weight study have cast doubt on the potential for reduction in heart disease and diabetes, we need to pause before adopting these cost figures. Many would love to find the holy grail for weight loss because we cannot forget that, despite the controversy that arose in late November 2004 when the Centers for Disease Control and Prevention admitted it had overestimated the number of deaths attributable to obesity, 365,000 Americans die annually from obesity, which is $15.2 \%$ of total U.S. deaths. ${ }^{33}$ Obesity is the second leading actual cause of death and, coming full circle, we also arrive back at tobacco-the leading cause of death in the United States (435,000 Americans, 18.1\% of total annual U.S. deaths). The statistics cannot be ignored; tobacco abuse and obesity are the top 2 modifiable behavioral risk factors for death in the United States, and we are eager for any potential tool to save lives.

The absolute benefit of medications for smoking cessation or weight-loss reduction is small, invoking the lyrics of the popular song by MC Hammer, "U Can't Touch This" with pharmacotherapy alone. ${ }^{34}$ The pharmacoeconomic benefits may be established for tobacco cessation but are less clear for weight-loss medications. The average weight loss in the LOSE Weight study at 6 months was $6.8 \mathrm{~kg}$ (6.3\%) for the drug group versus $3.1 \mathrm{~kg}$ (3.0\%) for the nondrug group, an absolute difference of $3.7 \mathrm{~kg}$, or about 3\% of baseline body weight for sibutramine compared with behavioral modification alone. More concrete evidence of improvement in cardiovascular morbidity or mortality is necessary to justify population-based coverage of weight-loss medications.

Now is the time to "pump up the volume" and advocate for MCOs to cover the costs for tobacco cessation medication when used in conjunction with behavior modification interventions. Until there are data that show that weight-loss medication improves health beyond dropping 3\% of baseline body weight, the decision to provide universal coverage needs to be deferred.

Frederic R. Curtiss, PhD, RPh, CEBS Editor-in-Chief fcurtiss@amcp.org

Brian K. Crownover, MD, FAAFP, Lt. Col., MC, USAF Associate Editor bcrownover@amcp.org 


\section{ACKNOWLEDGMENTS}

The opinions and assertions contained herein are the private views of the authors and are not to be construed as official or as reflecting the views of any organization, including the U.S. Air Force medical department or the U.S. Air Force.

\section{DISCLOSURES}

The authors disclose no potential bias or conflict of interest relating to this editorial

\section{REFERENCES}

1. Centers for Medicare and Medicaid Services. Medicare Chronic Care Improvement Program—national sample file of Medicare beneficiaries. Available at: http://www.cms.hhs.gov/medicarereform/ccip/Readme.pdf. Accessed June 12, 2005.

2. American Lung Association. Smokers not concerned about developing 4th leading cause of death, yet more than half experience symptoms once a week. Available at: http://www.lungusa.org/site/apps/nl/content2.asp?c= dvLUK9O0E\&b=34893\&ct=294905\&notoc $=1$. Accessed June 12, 2005

3. Tinkelman D, Nordyke RJ, Isonaka S, et al. The impact of COPD on longterm disability costs. J Manag Care Pharm. 2005;11(1):25-32.

4. Ruchlin HS, Dasbach EJ. An economic overview of chronic obstructive pulmonary disease. Pharmacoeconomics. 2001;19(6):623-42.

5. Maher K. Companies are closing doors on job applicants who smoke. Wall Street Journal. December 21, 2004:B6.

6. Parekh R. Companies fight tobacco use to lower health care costs. Bus Insur. February 28, 2005:4

7. Parekh R. States hit public employees with smoking surcharge. Bus Insur. May 23, 2005:3, 28.

8. CMS Media Affairs. Medicare will help beneficiaries quit smoking-new proposed coverage for counseling as Medicare shifts focus to prevention. Available at: http://www.os.dhhs.gov/news/press/2004pres/20041223a.html. Accessed December 31, 2004.

9. Geisel J. FSA money can fund smoking cessation: IRS. Bus Insur. June 28, 1999:2.

10. Anonymous. Statins will have "preventive" status under health savings accounts. Green Sheet. August 9, 2004:4

11. Wyeth-Ayerst Prescription Drug Benefit Cost and Plan Design Survey Report. 2000 ed. Tempe, AZ: Pharmacy Benefit Management Institute; 2000.

12. Aventis Managed Care Digest Series 2004. HMO-PPO/Medicare-Medicaid Digest. Sanofi-Aventis; 2004

13. Treating tobacco use and dependence-clinical practice guideline. U.S. Department of Health and Human Services, Public Health Service; June 2000. Available at: http://www.surgeongeneral.gov/tobacco/treating_tobacco_use.pdf Accessed June 16, 2005

14. Silagy C, Lancaster T, Stead L, Mant D, Fowler G. Nicotine replacement therapy for smoking cessation. Cochrane Database Syst Rev. 2004;3:CD000146. DOI:10.1002/14651858.CD000146.pub2.

15. Hughes JR, Stead LF, Lancaster T. Antidepressants for smoking cessation Cochrane Database Syst Rev. 2004:4:CD000031. DOI:10.1002/14651858. CD000031.pub2

16. Prochazka AV, Kick S, Steinbrunn C, et al. A randomized trial of nortriptyline combined with transdermal nicotine for smoking cessation. Arch Intern Med. 2004;164:2229-33

17. Simon JA, Duncan C, Carmody TP, Hudes ES. Bupropion for smoking cessation. Arch Intern Med. 2004:164:1797-803.

18. Kaper J, Wagena EJ, Severens JL, Van Schayck CP. Healthcare financing systems for increasing the use of tobacco dependence treatment. Cochrane Database Syst Rev. 2005;1:CD004305. DOI:10.1002/14651858.

CD004305.pub2.
19. Cromwell J, Bartosch WJ, Fiore MC, Hasselblad V, Baker T. Cost-effectiveness of the clinical practice recommendations in the AHCPR guideline for smoking cessation. Agency for Health Care Policy and Research. JAMA. 1997; 278(21):1759-66

20. Curry SJ, Grothaus LC, McAfee T, Pabiniak C. Use and cost effectiveness of smoking-cessation services under four insurance plans in a health maintenance organization. N Engl J Med. 1998;339(10):673-79.

21. National Committee for Quality Assurance. The state of health care quality: 2004. Industry trends and analysis. Available at: http://www.ncqa.org/communications/SOMC/SOHC2004.pdf. Accessed June 12, 2005.

22. Sinclair HK, Bond CM, Stead LF. Community pharmacy personnel interventions for smoking cessation. Cochrane Database Syst Rev. 2004;1: CD003698. DOI:10.1002/14651858.CD003698.pub2.

23. Snow V, Barry P, Fitterman N, et al. Pharmacologic and surgical management of obesity in primary care: a clinical practice guideline from the American College of Physicians. Ann Intern Med. 2005;142(7):525-31

24. Li Z, Maglione M, Tu W, et al. Meta-analysis: pharmacologic treatment of obesity. Ann Intern Med. 2005;142(7):532-46.

25. Gadde KM, Meta-analysis of pharmacologic treatment of obesity utilised irrelevant data. Ann Intern Med. 2005; May 3 (electronic letter). Available at: http://www.annals.org/cgi/eletters/142/7/532\#1565. Accessed June 12, 2005.

26. Croft H, Houser TL, Jamerson BD, et al. Effect on body weight of bupropion sustained-release in patients with major depression treated for 52 weeks. Clin Ther. 2002;24:662-72.

27. Malone DC, Raebel, MA, Poreter JA, et al. Cost-effectiveness of sibutramine in the LOSE Weight study: evaluating the role of pharmacologic weight-loss therapy within a weight management program. J Manag Care Pharm. 2005;11(6):458-68.

28. Porter JA, Raebel MA, Conner DA, et al. The Long-term Outcomes of Sibutramine Effectiveness on Weight (LOSE Weight) study: evaluating the role of drug therapy within a weight management program in a group-model health maintenance Organization. Am J Manag Care. 2004;10:369-76.

29. Jadad AR, Moore RA, Carroll D, et al. Assessing the quality of reports of randomized clinical trials: is blinding necessary? Control Clin Trials. 1996 17(1):1-12.

30. O'Meara S, Riemsma R, Shirran L, Mather L, ter Riet G. The clinical effectiveness and cost-effectiveness of sibutramine in the management of obesity: a technology assessment [review]. Health Technol Assess. 2002;6(6):1-97.

31. Avenell A, Broom J, Brown TJ, et al. Systematic review of the long-term effects and economic consequences of treatments for obesity and implications for health improvement [review]. Health Technol Assess. 2004;8(21):iii-iv, 1-182.

32. Warren E, Brennan A, Akehurst R. Cost-effectiveness of sibutramine in the treatment of obesity. Med Decis Making. 2004;24(1):9-19.

33. Mokdad AH, Marks JS, Stroup DF, Gerberding JL. Actual causes of death in the United States, 2000. JAMA. 2004;291(10):1238-45. Erratum in JAMA. 2005;293(3):293-94.

34. MC Hammer. "U Can't Touch This." Available at:

http://www.metrolyrics.com/lyrics/42863/MC_Hammer/U_Can't_Touch_This/. Accessed June 12, 2005.

\section{Letters to the Editor}

JMCP welcomes letters that serve to clarify subjects published in previous issues of the Journal or regarding subject matter of interest to managed care pharmacists. Letters in JMCP are not peer reviewed but are subjected to editorial review. Letters should be prepared in a word processing program, preferably Microsoft Word, and submitted electronically at jmcp.msubmit.net. See www.amcp.org for details. 